\title{
Crank-Nicolson discretization scheme and lattice fermions
}

\section{Artan Boriçi*i}

Physics Department University of Tirana Blvd. King Zog I Tirana - Albania

E-mail: boriciefshn.edu.al

\begin{abstract}
It is well known that Crank-Nicolson discretization scheme is second order accurate in time. Its application in the case of the Wilson operator in 3-space leads to a lattice theory which is second order accurate in time. Applying it in the case of minimally doubled Hamiltonian kernels one obtains chiral fermions with preserved unitarity. Finally, the scheme can be used to lift the doubler degeneracy of the naive lattice Hamiltonian kernel with the ground state describing a single Weyl fermion.
\end{abstract}

The 32nd International Symposium on Lattice Field Theory

23-28 June, 2014

Columbia University New York, NY

* Speaker.

${ }^{\dagger}$ A footnote may follow. 


\section{Introduction}

In lattice gauge theory it is important to have full control of discretization errors when taking the continuum limit. Therefore, lattice actions with smaller errors are preferred for simulation purposes. While in the gauge sector it is straightforward to have discretization errors of the second order in lattice spacing, the fermion sector is fundamentally more difficult. This can be illustrated by naive fermions, which in momentum space are defined by the Dirac operator:

$$
D_{N}(p)=\frac{i}{a} \sum_{\mu} \gamma_{\mu} \sin a p_{\mu}
$$

with $a$ being the lattice spacing. This operator has second order discretization errors in lattice spacing. However, it has 16 zeros located at the corners of the Brillouin zone which describe 16 degenerate flavors of Dirac fermions. The doublers can be given a mass at the order of cutoff by breaking explicitly the chiral symmetry, as proposed by Wilson many years ago [1]:

$$
a D_{W}(p)=i \sum_{\mu} \gamma_{\mu} \sin a p_{\mu}+r \sum_{\mu}\left(1-\cos a p_{\mu}\right)
$$

with $r$ being a real parameter. This way, the chiral symmetry is lost and discretization errors are of order $O(a)$. Moreover, in the presence of gauge fields, the quark mass gets additive renormalization terms. This difficulty is rooted to the impossibility of defining a single flavor of chiral fermions on the lattice [2]. However, it is possible to define two flavors of chiral fermions, as proposed some time ago by Karsten and Wilczek [4, 5]:

$$
a D_{K W}(p)=i \gamma_{4}\left[\sin a p_{4}+\lambda \sum_{k}\left(1-\cos a p_{k}\right)\right]+i \vec{\gamma} \sin a \vec{p},
$$

for $\lambda>1 / 2$. More recently, Creutz proposed to generalize the graphene structure in four dimensions obtaining in this way two flavors for Dirac fermions [6]. This theory can be reformulated on orthogonal lattices with the lattice Dirac operator resembling the Wilson operator by formally substituting the $r$ parameter in each direction with another set of gamma matrices $\gamma_{\mu}{ }^{\prime}$ multiplied by $i[7]$ :

$$
a D_{B C}(p)=i \sum_{\mu} \gamma_{\mu} \sin a p_{\mu}+\sum_{\mu} i \gamma_{\mu}{ }^{\prime}\left(\cos a p_{\mu}-1\right) .
$$

Such fermions have $O(a)$ errors, but more importantly they violate hypercubic symmetry and therefore the unitarity of the theory. It is the aim of this paper to define minimally doubled fermions with preserved unitarity by means of a new discretization scheme. This scheme allows also the formulation of two flavors of Wilson fermions in space which are second accurate in time.

\section{Crank-Nicolson discretization scheme}

When discretizing parabolic equations like the imaginary time Schrödinger equation:

$$
\partial_{t} \psi(t, x)=H \psi(t, x), \quad \psi(0, x)=\psi_{o}(x)
$$


one is essentially interested in approximating the exponential of the Hamiltonian operator which enters the wave function at positive times:

$$
\psi(t, x)=e^{t H} \psi(0, x), \quad t>0 .
$$

Traditional integration schemes include the forward Euler scheme, a finite difference scheme on a time lattice with lattice spacing $a$ :

$$
\psi(t+a, x)=(\mathbb{1}+a H) \psi(t, x),
$$

as well as the Crank-Nicolson scheme [3]:

$$
\psi(t+a, x)=\frac{\mathbb{1}+\frac{a}{2} H}{\mathbb{1}-\frac{a}{2} H} \psi(t, x) .
$$

While the latter is obtained from Euler scheme by averaging Hamiltonian contributions of adjacent time slices:

$$
H \psi(t, x) \rightarrow \frac{H}{2}[\psi(t, x)+\psi(t+a, x)],
$$

it has only $O\left(a^{2}\right)$ errors in expense of one inversion per time step.

\section{Crank-Nicolson lattice fermions}

The situation is slightly different when periodic boundary conditions apply as it is often the case for lattice fermions. Using a generic Hamiltonian kernel in 3-space, $H(\vec{p})$, the momentum space Dirac operator:

$$
a D(p)=\gamma_{4}\left(e^{i a p_{4}}-1\right)+\frac{1}{2} \gamma_{4} H(\vec{p})\left(e^{i a p_{4}}+1\right),
$$

has first order lattice spacing errors. However, the dispersion relation of the theory:

$$
4 \sin ^{2} \frac{a p_{4}}{2}+H(\vec{p})^{2} \cos ^{2} \frac{a p_{4}}{2}=0
$$

has order $O\left(a^{2}\right)$ errors in the continuum limit. While these errors are shared with naive fermions, the theory has half as many doublers. This property makes the Crank-Nicolson scheme a useful tool in defining lattice fermions with smaller discretization errors.

\subsection{Wilson fermions in 3-space}

The number of flavors of the operator (3.1) can be reduced to one if the Wislon discretization scheme is used in 3-space, thus obtaining the operator:

$$
a D_{W}^{(+)}(p)=\gamma_{4}\left(e^{i a p_{4}}-1\right)+\frac{1}{2}\left[i \vec{\gamma} \sin a \vec{p}+r \sum_{k}\left(1-\cos a p_{k}\right)\right]\left(e^{i a p_{4}}+1\right),
$$

where $r$ is the usual Wilson parameter, which we take here to be positive. It is easy to see that continuum limit of the dispersion relation of the theory:

$$
4 \sin ^{2} \frac{a p_{4}}{2}+\left\{\sin ^{2} a \vec{p}+\left[r \sum_{k}\left(1-\cos a p_{k}\right)\right]^{2}\right\} \cos ^{2} \frac{a p_{4}}{2}=0 .
$$


has order $O\left(a^{2}\right)$ errors along the time direction. Using the momentum space propagator of the theory:

$$
G_{W}^{(+)}(p)=\frac{e^{-i a p_{4} / 2}}{2 i \gamma_{4} \sin \frac{a p_{4}}{2}+\left[i \vec{\gamma} \sin a \vec{p}+r \sum_{k}\left(1-\cos a p_{k}\right)\right] \cos \frac{a p_{4}}{2}},
$$

and contour integration, it is straightforward to show that the theory is unitary. ${ }^{1}$ Note however, that time slices of propagators are shifted by a half lattice spacing. The broken parity resulted in this way can be restored in a two flavor theory defined by the Dirac operator:

$$
\begin{gathered}
D_{W}(p)=\left[\begin{array}{cc}
0 & D_{W}^{(+)}(p) \\
D_{W}^{(-)}(p) & 0
\end{array}\right], \\
a D_{W}^{(-)}=\gamma_{4}\left(1-e^{-i a p_{4}}\right)+\frac{1}{2}\left(e^{-i a p_{4}}+1\right)\left[i \vec{\gamma} \sin a \vec{p}+r \sum_{k}\left(1-\cos a p_{k}\right)\right] .
\end{gathered}
$$

Note also that $D$ is $\gamma_{5}$-symmetric and benefits from a smaller additive renormalization of the quark mass.

\subsection{Minimally doubled fermions with manifest unitarity}

A remarkable property of Crank-Nicolson discretization scheme is the fact that the unitarity of the theory is manifest. This property can be exploited in order to define minimally doubled fermions which preserve unitarity at a finite lattice spacing. The Hamiltonian kernel of the theory:

$$
H(\vec{p})=i \gamma_{4} \sum_{k}\left[\gamma_{k} \sin a p_{k}+\gamma_{k}^{\prime}\left(\cos a p_{k}-1\right)\right]
$$

satisfying the condition:

$$
\sum_{k} \gamma_{k}=\sum_{k} \gamma_{k}{ }^{\prime}
$$

has two zeros, one at the origin and the other one at $(\pi / 2, \pi / 2, \pi / 2)$. This way, the corresponding Dirac operator:

$$
a D_{+}(p)=\gamma_{4}\left(e^{i a p_{4}}-1\right)+\frac{i}{2} \sum_{k}\left[\gamma_{k} \sin a p_{k}+\gamma_{k}{ }^{\prime}\left(\cos a p_{k}-1\right)\right]\left(e^{i a p_{4}}+1\right),
$$

is chirally symmetric and unitary. ${ }^{2}$ Note that the cubic symmetry in 3-space remains broken even in this case. However, it is easy to see that the four flavor theory:

$$
\begin{gathered}
D(p)=\left[\begin{array}{cc}
0 & D_{+}(p) \\
D_{-}(p) & 0
\end{array}\right], \\
a D_{-}=\gamma_{4}\left(1-e^{-i a p_{4}}\right)+\frac{i}{2}\left(e^{-i a p_{4}}+1\right) \sum_{k}\left[\gamma_{k} \sin a p_{k}+\gamma_{k}{ }^{\prime}\left(\cos a p_{k}-1\right)\right],
\end{gathered}
$$

\footnotetext{
${ }^{1}$ See Appendix A for the proof.

${ }^{2}$ The proof is identically the same as in the case of Wilson fermions in 3-space.
} 
is $\gamma_{5}$-symmetric. The definition of the action is completed by defining the new set of gamma matrices:

$$
\left(\begin{array}{l}
\gamma_{1}^{\prime} \\
\gamma_{2}^{\prime} \\
\gamma_{3}^{\prime}
\end{array}\right)=\left(\begin{array}{lll}
\alpha_{11} & \alpha_{12} & \alpha_{13} \\
\alpha_{21} & \alpha_{22} & \alpha_{23} \\
\alpha_{31} & \alpha_{32} & \alpha_{33}
\end{array}\right)\left(\begin{array}{l}
\gamma_{1} \\
\gamma_{2} \\
\gamma_{3}
\end{array}\right)
$$

where $\alpha$ is an orthogonal matrix. In appendix B it is shown that $\gamma_{5}{ }^{\prime}=\operatorname{det} \alpha \gamma_{5}$. Hence, the chiral symmetry it is flavored as expected.

\subsection{Weyl fermions via the doubler degeneracy splitting}

A possible way to define a single chiral fermion and evade the Nielsen-Ninomiya theorem [2] is to lift the degeneracy of the doublers. The naive Weyl operator, which is based on the CrankNicolson discretization scheme,

$$
a \mathscr{W}^{\prime}(p)=e^{i a p_{4}}-1+\frac{1}{2} \vec{\sigma} \sin a \vec{p}\left(e^{i a p_{4}}+1\right),
$$

describes a quartet of chirality pairs with continuum limit energies:

$$
E(\vec{p})= \pm\left\{\begin{array}{l}
\sigma_{1} p_{1}+\sigma_{2} p_{2}+\sigma_{3} p_{3} \\
\sigma_{1} p_{1}+\sigma_{2} p_{2}-\sigma_{3} p_{3} \\
\sigma_{1} p_{1}-\sigma_{2} p_{2}+\sigma_{3} p_{3} \\
\sigma_{1} p_{1}-\sigma_{2} p_{2}-\sigma_{3} p_{3}
\end{array}\right.
$$

or in compact form:

$$
E_{\vec{p}}(\vec{p})=\cos p_{1}^{*} \sigma_{1} p_{1}+\cos p_{2}^{*} \sigma_{2} p_{2}+\cos p_{3}^{*} \sigma_{3} p_{3},
$$

with $\vec{p}^{*}$ being one of the corners of the three-dimensional Brillouin zone. Adding an imaginary Wilson term in 3-space, the new operator:

$$
a \mathscr{W}(p)=e^{i a p_{4}}-1+\frac{1}{2}\left[\vec{\sigma} \sin a \vec{p}+i r \sum_{k}\left(1-\cos a p_{k}\right)\right]\left(e^{i a p_{4}}+1\right),
$$

describes a quartet of chirality pairs with continuum limit energies:

$$
E_{\vec{p} *}(\vec{p})=\frac{\cos p_{1}^{*} \sigma_{1} p_{1}+\cos p_{2}^{*} \sigma_{2} p_{2}+\cos p_{3}^{*} \sigma_{3} p_{3}}{1+r^{2} n_{\vec{p}}^{2}},
$$

where the operator:

$$
n_{\vec{p}} *=\frac{1}{2} \sum_{k}\left(1-\cos a p_{k}^{*}\right),
$$

takes the integer values $0,1,2,3$ [8]. This way, the ground state of the theory describes a negative chirality chiral fermion at $n_{\vec{p}} *=3$ with the positive chirality partner being at the highest energy state at $n_{\vec{p}}{ }^{*}=0$. Intermediate states at $n_{\vec{p}}{ }^{*}=1,2$ have opposite chiralities and are three-fold degenerate. Since the operator $n_{\vec{p}}$ gets additive renormalization terms in the interacting theory, the chiral anomaly may survive in this case [8]. 


\section{A. Källén-Lehmann form of Crank-Nicolson lattice fermions}

The Crank-Nicolson fermion operator can be written in the form:

$$
D(p)=\gamma_{4} e^{i p_{4} / 2}\left[i 2 \sin \frac{p_{4}}{2}+H(\vec{p}) \cos \frac{p_{4}}{2}\right],
$$

where:

$$
H(\vec{p})=\gamma_{4}\left[h_{4}(\vec{p})+i \vec{\gamma} \vec{h}(\vec{p})\right],
$$

is the Hamiltonian kernel of the theory with $h_{\mu}(\vec{p}), \mu=1,2,3,4$ being a real vector field. ${ }^{3}$ Therefore, the quantum theory has a real energy spectrum. The negative energy norms are excluded if the propagator of the theory admits the Källén-Lehmann form:

$$
\mathscr{G}(t, \vec{x})=\int_{0}^{\infty} d E \int_{-\pi / a}^{\pi / a} \frac{d^{3} \vec{p}}{(2 \pi)^{3}} e^{-E t+i \vec{p} \vec{x}} \rho(E, \vec{p}), \quad t>0,
$$

where the spectral density $\rho(E, \vec{p})$ is positive semidefinite, i.e.:

$$
\bar{\psi} \rho(E, \vec{p}) \psi \geq 0
$$

for any value of the fermion field $\psi$. The fermion propagator of the Crank-Nicolson lattice theory can be written in the form:

$$
G(p)=e^{-i p_{4} / 2} \frac{-i 2 \sin \frac{p_{4}}{2}+H(\vec{p}) \cos \frac{p_{4}}{2}}{4 \sin ^{2} \frac{p_{4}}{2}+\omega(\vec{p})^{2} \cos ^{2} \frac{p_{4}}{2}} \gamma_{4},
$$

where $\omega(\vec{p})^{2}=\sum_{\mu} h_{\mu}(\vec{p})^{2}$. From here position space propagator of the infinite box:

$$
\mathscr{G}(t+a / 2, \vec{x})=\int_{-\pi / a}^{\pi / a} \frac{d^{4} p}{(2 \pi)^{4}} e^{i p_{4} t+i \vec{p} \vec{x}} \frac{-i 2 \sin \frac{p_{4}}{2}+H(\vec{p}) \cos \frac{p_{4}}{2}}{4 \sin ^{2} \frac{p_{4}}{2}+\omega(\vec{p})^{2} \cos ^{2} \frac{p_{4}}{2}} \gamma_{4}
$$

is evaluated using the contour integration with respect to $p_{4}$. Using the residue theorem one finds:

$$
\mathscr{G}(t+a / 2, \vec{x})=\int_{0}^{\infty} d E \int_{-\pi / a}^{\pi / a} \frac{d^{3} \vec{p}}{(2 \pi)^{3}} \frac{e^{-E t+i \vec{p} \vec{x}}}{4 \tanh \frac{E}{2}}\left[2 \sinh \frac{E}{2}+H(\vec{p}) \cosh \frac{E}{2}\right] \gamma_{4}, \quad t>-a / 2,
$$

where:

$$
4 \tanh ^{2} \frac{E}{2}=\omega(\vec{p})^{2} .
$$

Since the eigenvalues of the matrix $\omega(\vec{p})+H(\vec{p})$ are the twice degenerated values of zero and $2 \omega(\vec{p})$, the bilinear form:

$$
\bar{\psi}\left[2 \sinh \frac{E}{2}+H(\vec{p}) \cosh \frac{E}{2}\right] \gamma_{4} \psi
$$

is positive semidefinite. Hence, the negative norms of the quantum theory are thus excluded.

\footnotetext{
${ }^{3}$ It is assumed that $h_{4}(\vec{p})$ vanishes in the continuum limit.
} 


\section{B. Chirality of the unitary minimally doubled fermions}

If gamma matrices are given in the chiral representation:

$$
\gamma_{k}=\left(\begin{array}{cc}
{ }^{-i \sigma_{k}} \\
i \sigma_{k}
\end{array}\right), \quad \gamma_{4}=\left(\begin{array}{ll} 
& \mathbb{1} \\
\mathbb{1} &
\end{array}\right),
$$

then the definition of the new set of gamma matrices in (3.5) implies that the same relation holds in terms of Pauli matrices, i.e. $\sigma^{\prime}=\alpha \sigma$. Using the Pauli matrices property:

$$
\sigma_{k} \sigma_{l}=\delta_{k l}+i \varepsilon_{k l m} \sigma_{m}
$$

and the fact that the matrix $\alpha$ is orthogonal, it is easy to show that:

$$
\sigma_{1}{ }^{\prime} \sigma_{2}{ }^{\prime} \sigma_{3}{ }^{\prime}=\sum_{j k l} \alpha_{1 j} \alpha_{2 k} \alpha_{3 l} \sigma_{j} \sigma_{k} \sigma_{l}=i \operatorname{det} \alpha
$$

This way:

$$
\gamma_{1}{ }^{\prime} \gamma_{2}{ }^{\prime} \gamma_{3}{ }^{\prime}=\left(\begin{array}{cc}
0 & -i \sigma_{1}{ }^{\prime} \sigma_{2}{ }^{\prime} \sigma_{3}{ }^{\prime} \\
i \sigma_{1}{ }^{\prime} \sigma_{2}{ }^{\prime} \sigma_{3}{ }^{\prime} & 0
\end{array}\right)=\operatorname{det} \alpha\left(\begin{array}{rr}
0 & \mathbb{1} \\
-1 & 0
\end{array}\right),
$$

and therefore $\gamma_{5}{ }^{\prime}=\gamma_{1}{ }^{\prime} \gamma_{2}{ }^{\prime} \gamma_{3}{ }^{\prime} \gamma_{4}=\operatorname{det} \alpha \gamma_{5}$.

\section{References}

[1] K. G. Wilson in New Phenomena In Subnuclear Physics, ed. A. Zichichi, Plenum Press, New York, 1977.

[2] H.B. Nielsen, M. Ninomiya, Nucl.Phys.B185:20 (1981), Erratum-ibid.B195:541 (1982).

[3] J. Crank and P. Nicolson, Proc. Camb. Phil. Soc. 43 (1947) 50-67, repr. in Adv. Comp. Math. 6 (1996) 207-226.

[4] L. H. Karsten, Phys. Lett. B 104, 315 (1981).

[5] F. Wilczek, Phys.Rev.Lett.59:2397 (1987).

[6] M. Creutz, JHEP 0804, 017 (2008).

[7] A. Boriçi, Phys.Rev.D78:074504 (2008); PoS LATTICE2008:231 (2008).

[8] A. Boriçi, in preparation. 